\title{
A Cross-domain Authentication Method for Cloud Computing
}

\author{
Chen $\mathrm{Xu}$ and Jingsha $\mathrm{He}$ \\ School of Software Engineering \\ Beijing University of Technology Beijing 100124, China \\ xc_cn@foxmail.com,jhe@bjut.edu.cn
}

\begin{abstract}
The use of security certificates under the Cloud environment is the foundation to establish mutual trust between the Cloud and the user. In this paper, we propose an authentication method based on zero-knowledge proof and the mind of key escrow. With the method, authentication will not only satisfy the requirement anonymity and security but also can recover the real identity information in special circumstances with the cooperation of multiple parties. We will show that this proposed method is more suitable for promotion through analysis and comparison with an existing scheme.
\end{abstract}

Keywords: Cloud Computing, Security Certificate, Zero-knowledge Proof, Anonymity, Traceability

\section{Introduction}

Cloud computing is a computing model which can provide users with computing, storage and software application services through the network. Users under the cloud environment do not need to buy much hardware such as the infrastructure or expensive computing devices, thus reducing the cost for them. Users can get services through the network from the cloud and then pay the corresponding fees to the service provider [1]. Cloud services have a bright application prospect.

The development of cloud computing has been making provided services more and more important. Since a single cloud environment may not be able to meet the requirements of users, the use of services provided by external public clouds becomes necessary. Then, each cloud environment would have its own users and resources within different trust domains. To guard against the fake identity attack, clouds should only provide services to users whose identities have already been validated. We call the authentication by a user to access a private cloud domain authentication and that to access a public cloud cross-domain authentication.

Cross-domain authentication is the basis of connecting and operating distributed multi-domain systems. It is also the key to realize the security access between distributed and multi-domain systems. The purpose of cross-domain authentication is to allow users to access server resources in multiple domains transparently. The condition for cross-domain authentication is that the cloud already has formed independent domains and different domains only trust their own users. A user would complete authentication with its own domain authentication sever. If the user belongs to a foreign domain, the authentication server will need to carry out some extra communication with the home domain authentication server to authenticate the user.

The benefits of cross-domain authentication for users include reducing the number of log-ins and increasing the efficiency because the service provider would reduce the number of regular authentications. It can also reduce the number of user passwords in the network 
transmission and thus reduce the security risk. Cross-domain authentication for the entire domains in the network would realize the interconnectivity between each other and bring more benefits such as flexibility, security, management and cost improvement.

\section{Related Work}

Cross-domain authentication provides users with convenience. At the same time, it may lead to some potential safety hazard when a user uses a single identity to access different domain services due to the risk that malicious users can access records and sessions. Therefore, how to hide the real identity of the users in the process of authentication becomes a critical issue in cross-domain authentication. In addition, cross-domain authentication should satisfy the requirement of anonymity which means that the domain authentication server and other users cannot know the true identity of legitimate users. At the same time, because a user may not provide true identity information during cross-domain authentication, the authentication server requires sufficient evidence to prove that the user is trusted by its own domain. There are also some special situations (such as judicial intervention) which need to track the real information of anonymous users, so the random identification algorithm is difficult to be widely used in practice. Therefore, cross-domain authentication also needs to satisfy a certain degree of traceability.

Both literatures [2,3] use attribute certificates and sign the general environment of secret technology to achieve cross-domain authentication, but there is no guarantee on anonymity. In literature [4], the identity is based on a single sign-on technology alliance technology and a cross-domain authentication scheme is designed to protect the user's anonymity. But it needs too much time for the communication between different domains.

Literature [5] proposes a provable security authentication protocol based on a convertible agent signing and uses the authentication protocol to achieve one-time authentication. A user can go through the authentication directly after registration, but this mechanism cannot track the user's cross-domain access records and the user's real information and the safety of the signature are dependent on the private cloud. Hence, leakage of customer data be will allow the attacker to use a fake ID to illegal use the resources. Literature [6] proposes an identity signature algorithm based on elliptic curve additive group. In the algorithm, the signature verification results of different users are some constants, but this kind of scheme similar to complete anonymity is not suitable for actual promotion.

Based on related work, we propose to design a kind of zero-knowledge proof based on RSA signature algorithm authentication scheme. The scheme provides different ways of identity authentication in in-domain and cross-domain access. At the same time, we introduce the threshold scheme to split the user information and store it with different authentication participants. Each participant cannot get the real information by itself. Our scheme can not only provide anonymity but also realize the authentication with traceability.

\section{The Signature Algorithm based on Zero-Knowledge Proof}

In early 1980s, Goldwasser put forward the concept of zero-knowledge proof. The basic concept is that certifier $P$ has some secret information and through a series of agreements, $P$ can prove to verifier $V$ that he knows this information, but doesn't need to provide any related information to $V$. This process of a series of agreement is called zero-knowledge proof protocol.

\subsection{Construction of the Parameters}


We assume that each user has a unique identity $I$. $I$ is converted to $J$ by using the hash function $H$, i.e., $J=H(I)$. We suppose that user $A$ has unique identity $I_{\mathrm{A}}$, corresponding to the hash value of $J_{\mathrm{A}}$. A trusted third party selects a pair of large prime numbers $p$ and $q$, calculates $n=p * q$, chooses $e$ such that $\operatorname{gcd}(e,(p-1) *(q-1))=1$, generates its own public key $(e, n)$ and the private key $d$, where $d=e^{-1} \bmod ((p-1),(q-1))$, then generates a secret parameter $S_{A}$ for user $A$, where $S_{A}=J_{A}^{-d} \bmod n$, $\left(1<J A<n, \operatorname{gcd}\left(J_{A},(p-1) *(q-1)\right)=1\right)$.

\subsection{Creation of a Signature}

$A$ sends message $M$ and the signature to $B$, The signature is generated by using $S_{A}$.

(1) $A$ chooses a random number $r(1<\mathrm{r}<(\mathrm{n}-1))$ and calculates $x=x^{e} \bmod n$.

(2) $A$ calculates $t=H(M, x)(0<t<e)$, where $M$ is the message that needs to be signed.

(3) $A$ calculates $T=r * S_{A}^{t} \bmod n$ and set the signature with $\left(t, T, I_{A}\right)$.

\subsection{Verification of the Signature}

$B$ will verify the signature when it received the message.

(1) $B$ calculates $J_{A}=H\left(I_{A}\right)$.

(2) $B$ calculates $T^{\prime}=T^{e} * J_{A}^{t} \bmod n$ and then calculates $t^{\prime}=H\left(M, T^{\prime}\right)$.

(3) $B$ compare $t$ to $t^{\prime}:$ if $t=t^{\prime}$, then $B$ can believe that $A$ knows the secret parameter $S_{A}$, message $M$ is correct and confirms $A$ is a legitimate user.

\subsection{Certification}

(1) From $S_{A}=J_{A}^{-d} \bmod n$, deduce $J_{A}=S_{A}^{-e} \bmod n$, then get Formula 1:

$$
r^{e} * J_{A}^{t}=r^{e} * S_{A}^{-e t} \bmod n
$$

(2) From $x=x^{e} \bmod n$ and Formula 1, get Formula 2:

$$
r^{e} * J_{A}^{t} * S_{A}^{e t} \bmod n=x
$$

(3) From $T=r * S_{A}^{t} \bmod n$ and Formula 2, get Formula 3:

$$
T^{e} * J_{A}^{t} \bmod n=x
$$

(4) From $T^{\prime}=T^{e} * J_{A}^{t} \bmod n$ and Formula 3, deduce $T^{\prime}=x$, if $t=t^{\prime}$, then $H \quad\left(M, T^{\prime}\right)=H(M$, $x$ ), so the availability can be proved.

\section{Construction of the Scheme}

Suppose that there are two independent security domains $A$ and $B$, as shown in Figure 1. Each security domain has an authentication server to manage resources and the users in this domain. User $U$ belongs to domain $A$. Then, we name the authentication server in domain $A$ as $H A$ (home authenticator) and the authentication server in domain $B$ as $F A$ (foreign authenticator). $U$ has already registered with $H A$ in advance and $H A$ has sent authentication parameters to $U$, so $U$ can directly use the authentication parameters during verification when he accessed resources in domain $A$. When $U$ needs to access domain $B$, however, he will generate his own identity signature and send it to $F A$. $F A$ will set a temporary ID in domain $B$ for $U$. After the completion of verification of the signature information, $U$ can use new authentication parameters to access $B$. The basis of this scheme is that $H A$ and $F A$ mutually trust each other, share public key with each other and both of them use a sharing function $H$. 


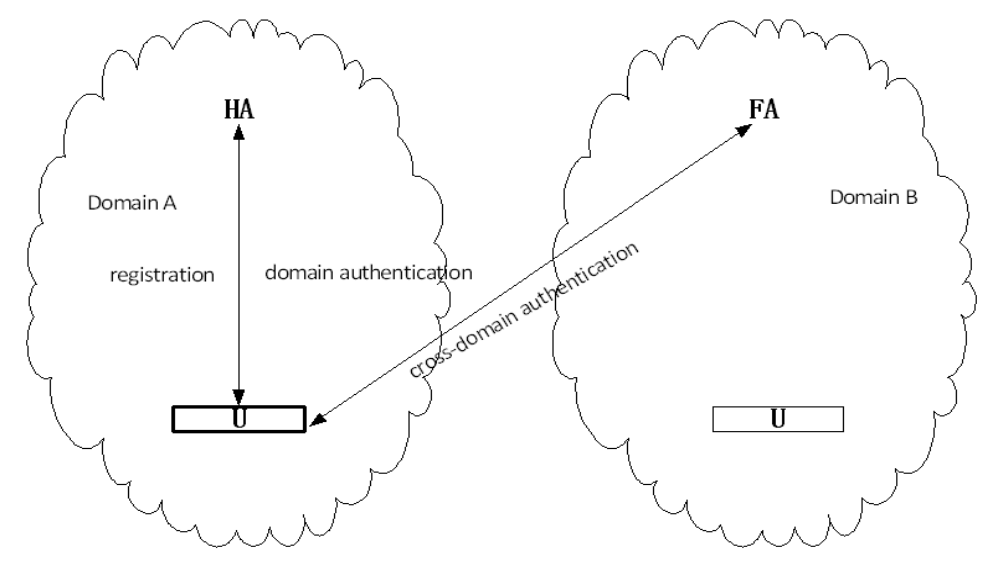

Figure 1. Construction of the Scheme

\section{Design of the Scheme}

The scheme includes four stages: system establishment, registration, domain authentication and cross-domain authentication. As shown in Figure 2, in the system establishment stage, $H A$ chooses its own system parameters. In the registration stage, $H A$ creates a temporary account for $U$ and sends the communication key and the identity certificate issued to $U$. When $U$ performs access in the domain, $A$ will go through the domain authentication directly. When $U$ performs access in domain $B, U$ will be verified by $F A$ during which $F A$ creates a temporary account within domain $B$ for $U$ and issues the communication key to $U$. When $U$ performs access again using the temporary account, it will only need to use the communication key to achieve authentication.

\subsection{System Establishment}

$H A$ sets safety parameters, i.e., it generates its own public key $(e, n)$, the private key $d$, chooses the hash function $H$ and publishes parameters $\{(e, n), H\}$.

\subsection{Registration}

1) $U$ sends the real identity information to $H A$.

2) $H A$ creates a temporary account for $U$, i.e., $I D_{\mathrm{U}}$. The period of validity of the account is $T, H A$ encrypts the real identity information by using $(e, n)$ and generates $I_{\mathrm{U}}$. $H A$ divides $I_{\mathrm{U}}$ into $k$ segments by using Shamir's $(t, k)$ threshold scheme and stores $t-1$ segments in the account as $I_{U} \mid t-1$ and chooses the other one segment as $I_{U} \mid 1$, then $H A$ calculates identity certificate $C_{U}=\left\{I_{U} \mid 1, T\right\}^{-d} \bmod n$.

3) $H A$ calculates $J_{U}=H\left(C_{U}\right)$ and $S_{U}=J_{U}^{-d} \bmod n$, then it establishes account index $\left\{I D_{U}\right.$, $\left.S_{\mathrm{U}}, I_{\mathrm{U}} \mid \mathrm{t}-1, \mathrm{~T}\right\}$.

4) $H A$ sends account message $\left\{I D_{\mathrm{U}}, C_{\mathrm{U}}, S_{\mathrm{U}}\right\}$ to $U, I D_{\mathrm{U}}$ is the legal identity which $U$ can use in domain $A$ within the $T$ time period of availability. 


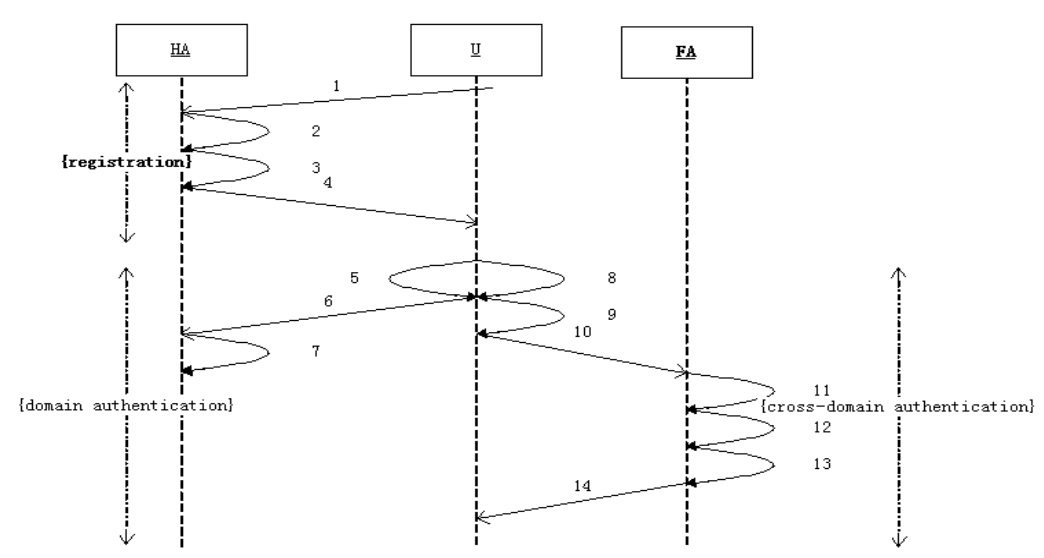

Figure 2. Procedure of the Scheme

\subsection{Domain Authentication}

5) $U$ calculates $J_{U}^{\prime}=H\left(C_{U}\right)$, then it generates message $N$ by encrypting $J_{\mathrm{U}}$ ' through using $S_{\mathrm{U}}$.

6) $U$ sends verification information $\left\{I D_{U}, U, N, T_{U}\right\}$ to $H A$, where $T_{\mathrm{U}}$ is the current time.

7) $H A$ gets the temporary account $I D_{\mathrm{U}}$ and decrypts $N$ by using $S_{\mathrm{U}}$ to get $J_{\mathrm{U}}$, then $H A$ calculates $J_{U}=S_{U}^{-e} \bmod n$. If $J_{\mathrm{U}}=J_{\mathrm{U}}$, then $H A$ can confirm that $U$ is a legitimate user, then $H A$ sets $S_{\mathrm{U}}$ as the communication key to establish communication with $U$.

\subsection{Cross-domain Authentication}

8) $U$ chooses a random number $r(1<r<(n-1))$, then it calculates $x=r^{e} \bmod n$ and generates message $M\left\{I D_{H A}, T_{U}, I D_{U}\right\}$.

9) $U$ calculates $t=H(M, x)(0<t<e), T=r * S_{u}^{t} \bmod n$ and generates signature sig $\{t, T$, $\left.C_{U}\right\}$.

10) $U$ sends $\operatorname{sig}\left\{t, T, C_{U}\right\}$ and $M$ to $F A$.

11) $F A$ gets $I D_{\mathrm{HA}}$ along with the parameters $\{(e, n), H\}$.

12) $F A$ calculates $\left\{I_{U} \mid 1, T\right\}=C_{U}^{-e} \bmod n$, gets time $T$, and then tries to verify the signature.

13) $F A$ calculates $J_{U}^{\prime}=H\left(C_{U}\right), T^{\prime}=T^{e} * J_{U}^{t} \bmod n, t^{\prime}=H\left(M, T^{\prime}\right)$, if $t=t^{\prime}$, then confirms that $U$ is a legitimate user.

14) $F A$ creates a temporary account for $U$ and sets the ID as $I D_{U}^{\prime}, F A$ chooses $S_{U}^{\prime}$ as the communication key, establishes account index $\left\{\operatorname{ID}_{U}^{\prime}, \mathrm{S}_{U}^{\prime}, \mathrm{C}_{\mathrm{U}}, \mathrm{T}\right\}$ and sends $\left\{\mathrm{ID}_{U}^{\prime}, \mathrm{S}_{U}^{\prime}\right\}$ to $U$. $F A$ then establishes communication with $U$ by using $S_{U}^{\prime}$.

\section{Analysis of the Scheme}

\subsection{Security Analysis}

The proposed scheme provides different ways of identity authentication for in-domain and cross-domain access. For in-domain access, the main security threats are session hijacking and middleman attacks. However, if the key which is defined in prior agreement to encrypt the communication is used, all these threats will become insignificant. For the to the cross-domain access, the main security threat comes from the fake identity. The proposed 
scheme solves this problem by introducing the zero-knowledge proof to make sure that $F A$ can verify the identity without acquiring the user information.

Authentication by $H A$ : $U$ generates message $N$ through encrypting $J_{\mathrm{U}}$ ' by using $S_{\mathrm{U}}$ after registration and then sends $N$ to $H A$. $H A$ gets the temporary account from $I D_{\mathrm{U}}$ and decrypts $N$ by using $S_{\mathrm{U}}$ to confirm that $U$ is a legitimate user. Since $S_{\mathrm{U}}$ is defined in a prior agreement, it is not possible to get the $S_{\mathrm{U}}$ through eavesdropping. Therefore, communication within the domain is secure.

Authentication by $F A$ : $U$ generates message $M$ and sends it to $F A$. $F A$ verifies the signature by using the zero-knowledge proof. If $F A$ can confirm that $U$ is a legitimate user, it will create a temporary account in domain $B$ for $U$. Since message $M$ contains a different timestamp, the randomness of message $M$ will make sure that $M$ can hardly be forged. Besides, $S_{\mathrm{U}}$ which is be used to generate the signature doesn't appear in the communication, so the eavesdropper cannot get $S_{\mathrm{U}}$ by eavesdropping. Therefore, the communication cross the domain is also secure.

\subsection{Anonymity and Traceability Analysis}

\subsubsection{Shamir (t, n) Threshold Scheme}

The scheme adopts the design idea of "secret sharing" [6] which has the advantage of sharing risks. The scheme works as follows.

Set the system parameters: We give a set of members $P=\{P 1, P 2 \ldots P n\}$, threshold value $t$ $(0<t<n)$ and trust center TA. TA selects a Galois field $G F(p)$ where $p$ is a large prime number. Each member of $P$ has its own public ID $x_{i}(i=1,2 \ldots n)$ and $x_{i} \in G F(p)$.

Distribute the subkeys: We select a random polynomial of degree $t$-1, i.e., $f(x)=$ $k+a_{1} x+a_{2} x^{2}+\ldots+a_{t-1} x^{t-1}\left(a_{i} \in G F(p), k \in G F(p)\right)$, where $a_{i}$ and $k$ are the sharing secret. Then we calculate $f\left(x_{i}\right)$ and send it to $P_{i}$ in order $(i=1,2 \ldots n) . f\left(x_{i}\right)$ is the subkey of $P_{i}$.

Recover the key: We choose $t$ members from $P$ and make them cooperate to get $\left(\left(x_{1}\right.\right.$, $\left.\left.f\left(x_{1}\right)\right) \ldots\left(x_{t}, f\left(x_{t}\right)\right)\right)$. We can thus reconstruct the polynomials by using Formula (4).

$$
\mathrm{f}(\mathrm{x})=\sum_{\mathrm{i}=1}^{\mathrm{t}} \mathrm{f}\left(\mathrm{x}_{\mathrm{i}}\right) \prod_{\substack{j=1 \\ j \neq i}}^{t} \frac{x-x_{i}}{x_{i}-x_{j}}
$$

Then, we can recover the sharing secret $k$ by using Formula (5).

$$
\mathrm{k}=\mathrm{f}(0)=\sum_{i=1}^{t} f\left(x_{i}\right) \prod_{\substack{j=1 \\ j \neq i}}^{t} \frac{-x_{i}}{x_{i}-x_{j}}
$$

We can see from Shamir's $(t, n)$ threshold scheme that it is not possible to recover the sharing secret $k$ while the number of cooperators within $P$ is less than $t$. If a member $P_{i}$ wants to get the polynomial $f(x)$ from its own subkey $f\left(x_{i}\right)$, the problem it will deal with is that the discrete logarithm of elliptic curve is an unsolved problem in mathematics.

\subsubsection{Anonymity Analysis}

After registration, the real identity information is encrypted and split into segments. The only credential for the user is the temporary account. According to the threshold scheme theorem, $H A$ holds the $t-1$ segments, $U$ and $F A$ both hold the same segment. None of them could recover the real identity information. Therefore, the real user cannot be associated with the user access records. Thus, the user can realize anonymity completely in both in-domain authentication and cross-domain authentication. 


\subsubsection{Traceability Analysis}

The records of the user to perform in-domain access and cross-domain access cannot be associated with the real identity information, thus meeting the requirement of anonymity. However, in some special circumstances, the real user information should be recovered. In this case, with the cooperation of multiple institutions, $H A$ can get all the segments so that it to recover the real identity information to achieve the purpose of tracking the user's access records.

\subsection{Efficiency Analysis}

The analysis on efficiency is performed on counting the number of periods for cross-domain identification. After comparing the proposed method to the scheme in literature [6], we can see that both schemes achieve identification anonymity and security but through different strategies as shown in Table 1.

\section{Table 1. Comparison of the Schemes}

\begin{tabular}{|c|c|c|c|c|c|}
\hline scheme & $\begin{array}{c}\text { Sign and Verify } \\
\text { (U/HA/FA) }\end{array}$ & $\begin{array}{c}\text { Encrypt and Decrypt } \\
\text { (U/HA/FA) }\end{array}$ & $\begin{array}{c}\text { Interactions } \\
(\mathrm{U}-\mathrm{FA} / \mathrm{FA}-\mathrm{HA})\end{array}$ & $\begin{array}{l}\bullet \text { An } \\
\text { onymity }\end{array}$ & $\begin{array}{l}\bullet \\
\text { aceability }\end{array}$ \\
\hline Literature [6] & $0 / 2 / 2$ & $0 / 1 / 1$ & $2 / 2$ & yes & no \\
\hline This & $1 / 0 / 1$ & $1 / 1 / 1$ & $2 / 0$ & yes & yes \\
\hline
\end{tabular}

In our scheme, the signature is generated by $U$. In the other scheme, however, $U$ needs to gets the signature from $H A$ first and then uses the received signature. Therefore, our scheme omits two information interactions and thus reduces the time for network communication. Besides, in the whole process of cross-domain identification, zero-knowledge proof is used in our scheme to identify the legality of the user by $F A$. Thus, a lot of bandwidth that needs to be consumed by cross-domain communication can be saved. In the registration and authentication process, all the entities that are participating in the communication are responsible for a part of the computation task, thus making full use of the computation resources, resulting in reducing the waiting time by other entities while an entity is conducting massive computation. In terms of anonymity, both schemes can achieve the goal. The difference is that our scheme ensures that $H A$ and $F A$ cannot obtain the user information alone. Moreover, our scheme provides traceability under specific circumstances. Therefore, our scheme is superior to the scheme in literature [6] in terms of both information sesurity and practical application.

\section{Conclusion}

To support extensive applications of cloud computing services, domain and cross-domain authentications place some special requirements along with changes in real applications. This paper proposed a scheme based on zero-knowledge proof and the mind of key escrow, both of which are applicable to both domain and cross-domain authentications that can meet the demands for establishing secure communication and improving user's anonymity. Compared to a current scheme, our scheme ensures the basic requirement of security. In addition, it cannot only reduce the computation overhead between communication entities that participate in the authentication, but also meet the need for obtaining users' real information under special circumstances. Therefore, it is more suitable to be used in practical applications.

\section{Acknowledgement}


The work in the paper has been supported by National Natural Science Foundation of China (61272500) and Beijing Natural Science Foundation (4142008).

\section{References}

[1] A. Weiss, "Computing in the Clouds", Network, vol. 11, (2007), pp. 16-25.

[2] D. G. Lee, S. Kang and D. H. Seo, "Authentication for single/multi domain in ubiquitous computing using certification", International Conference on Computational Science and Its Applications, Glasgow, UK, (2006), pp. 326-335.

[3] L. Yao, L. Wang and X. W. Kong, "An inter-domain authentication scheme for pervasive computing environment", Computers and Mathematics with Applications, vol. 59, no. 2, (2010), pp. 811-821.

[4] Y. Chan and S. Fleissner, "Single sign-on and key establishment for ubiquitous smart environments", International Conference on Computational Science and Its Applications, Glasgow, UK, (2006), pp. 406-415.

[5] T. F. Lee, T. Hwang and S. H. Chang, "Enhanced delegation-based authentication protocol for PCSs", IEEE Transactions on Wireless Communications, (2009).

[6] C. Y. Luo, S. W. Huo and H. Z. Xing, "Identity based cross-domain authentication scheme in pervasive computing environments", Journal on Communications, 09-0111-05, (2011).

[7] A. Shamir, "How to share a secret", Communications of the ACM, vol. 22, no. 11, (1979), pp. 612-613.

\section{Authors}

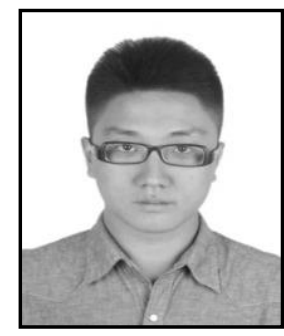

Chen Xu is currently a master's student in the School of Software Engineering at Beijing University of Technology in Beijing, China. His main research interests are software engineering and network security.

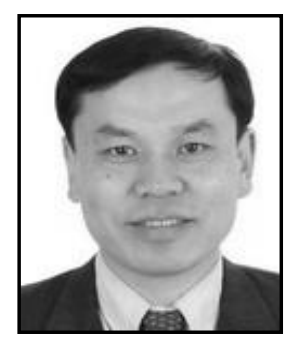

Jingsha He is currently a professor and Ph.D. advisor in the School of Software Engineering at Beijing University of Technology in Beijing, China. He received the B.S. degree in computer science from Xi'an Jiaotong University in Xi'an, China in 1982 and the M.S. and Ph.D. degrees from the University of Maryland at College Park in Maryland, U.S.A. in 1984 and 1990, respectively. Prior to joining Beijing University of Technology (BJUT) as an endowed professor of the City of Beijing in 2003, Prof. He worked for several hi-tech companies in the United States such as IBM Federal Systems, MCI Communications Corp., GRIC Communications, Inc. and Fujitsu Laboratories of America, Inc. where he carried out research and development in the areas of computer networking and information security. Prof. He's main research interests include computer and network security, network measurement and wireless communication protocols and security. His academic accomplishments include over 200 publications in scholarly journals and major international conferences, 12 U.S. patents and over 30 China patents, 22 software copyrights and 6 authored books. 